\title{
The role of repeat fine needle aspiration in the management of indeterminate thyroid nodules
}

\author{
Alborz Jooya', Joe Saliba', Audrey Blackburn', Michael Tamilia², Michael P. Hier ${ }^{1}$, Alex Mlynarek', \\ Véronique-Isabelle Forest ${ }^{1}$, Louise Rochon ${ }^{3}$, Anca Florea ${ }^{3}$, Hangjun Wang ${ }^{3}$ and Richard J. Payne ${ }^{1 *}$
}

\begin{abstract}
Background: Management decisions are not straightforward when the Ultrasound Guided Fine Needle Aspiration (USFNA) demonstrates a Bethesda score of either category III or IV, and a diagnostic hemi-thyroidectomy or a repeat USFNA (r-USFNA) could be performed. The aim of this study is to assess the effectiveness of r-USFNA in the management of indeterminate thyroid nodules by evaluating the likelihood of obtaining a definite diagnosis.

Methods: We reviewed the medical records of all patients with thyroid nodules between 2011 and 2015 at the Jewish General Hospital (Montreal, Canada). Three hundred fifty-one patients who had undergone a surgical procedure (hemi or total thyroidectomy) and a diagnosis of B3 or B4 on the primary USFNA (p-USFNA) were included in the study. Ninety-six of the included patients also had a repeat USFNA prior to the surgery.

Demographic data, type of procedure, and McGill Thyroid Nodule Score (MTNS) were obtained from the medical records. Malignancy rates were calculated based on the final surgical histopathology report.

Results: Upon r-USFNA, an average $76 \%$ of patients did not change Bethesda categories, $7.4 \%$ downgraded to a benign category. The results showed that, on an average $17.3 \%$ of patients with p-USFNA of B3 and $20 \%$ of patients with p-USFNA of B4, upgraded to a malignant or suspicious for malignancy category, thus changing the clinical management to total thyroidectomy. Our data demonstrates that r-USFNA facilitates choosing the correct surgery of total thyroidectomy in about $20 \%$ of nodules that have upgraded from B3/B4 to a more definite malignant category.

Conclusions: r-USFNA in patients with indeterminate diagnoses (B3 or B4) increases categorization into more definite categories. Approximately $20 \%$ of patients are found to have malignant thyroid nodules and suspicious for malignancy thyroid nodules upon repeating the biopsy, hence a diagnostic hemi-thyroidectomy was avoided and a more definitive surgery could be performed. Furthermore, repeat USFNA results in a fewer number of hemi-thyroidectomy and completion thyroidectomy procedures.
\end{abstract}

Keywords: Ultrasound Guided Fine Needle Aspiration, FNA, Bethesda, Repeat FNA

\footnotetext{
* Correspondence: rkpayne@sympatico.ca

${ }^{1}$ Department of Otolaryngology - Head and Neck Surgery - Jewish General Hospital, McGill University, 3755 Chemin de la Côte-Sainte-Catherine, Room E-903, Montréal H3T 1E2, QC, Canada

Full list of author information is available at the end of the article
} 


\section{Background}

Ultrasound Guided Fine Needle Aspiration (USFNA) is a cost-effective and highly reliable procedure used to evaluate and guide the management of thyroid nodules [1]. The Bethesda System for Reporting Thyroid Cytopathology (BSRTC) has resulted in standardization, reproducibility and improved clinical significance of thyroid USFNA. It is comprised of the following six categories: B1, non-diagnostic; B2, benign; B3, Atypia of undetermined significance or follicular lesion of undetermined significance; B4, (suspicious for) Follicular neoplasm; B5, Suspicious for malignancy; B6, malignant. Management decisions are not straightforward when the USFNA demonstrates a Bethesda score of either category III (B3) or IV (B4) [2-6]. In those instances, it is not uncommon for some patients to undergo diagnostic hemi-thyroidectomy to obtain a definitive diagnosis, while others prefer a more conservative approach and opt for a repeat USFNA (r-USFNA). However, none of these scenarios are ideal. A hemi-thyroidectomy is rarely the procedure of choice for a thyroid nodule: it is either unnecessary (in the event of a benign lesion) or inadequate (in the event of a malignant lesion). Likewise, the benefit of repeat USFNA is unclear with respect to its contribution to patient management. If the r-USFNA results in Bethesda II (B2), Bethesda V (B5) or Bethesda VI (B6), then management recommendations are more clear and the test is deemed to be useful. However, if the test yields a Bethesda I (B1), B3, or B4, then rUSFNA is not helpful. The aim of this study is to assess the effectiveness of repeat USFNA in the management of indeterminate thyroid nodules (B3 and B4) by evaluating the likelihood of obtaining a definite diagnosis. Moreover, this investigation assesses the usefulness of r-USFNA in the prevention of completion hemi-thyroidectomies in patients with initial B3 or B4 diagnoses.

\section{Methods}

A retrospective chart review of all patients with thyroid nodules classified by BSRTC who underwent a hemi- or total thyroidectomy between 2011 and 2015 at the Jewish General Hospital (Montreal, Canada) was performed. Three hundred fifty-one patients who had undergone a surgical procedure (hemi or total thyroidectomy) and a diagnosis of $\mathrm{B} 3$ or $\mathrm{B} 4$ on the primary USFNA (p-USFNA) were included in the study. Ninety-six of the included patients also had a repeat USFNA prior to the surgery. Demographic data, type of procedure, and McGill Thyroid Nodule Score (MTNS) were obtained from the medical records. Patients with missing medical data or a diagnosis other than B3 or B4 on p-USFNA were excluded from the study. Malignancy rates were calculated based on the final surgical histopathology report. Fine needle aspirations were performed by otolaryngologists or interventional radiologists under ultrasound guidance using 25 - or 27 -gauge needle with typically one to four passes.

The decision to undergo thyroidectomy in the patient population was influenced by a high McGill Thyroid Nodule Score (MTNS). MTNS was previously shown to accurately represent the risk of malignancy in a thyroid nodule, given it takes into account a variety of demographic and clinical risk factors in addition to the cytological features evident on USFNA [7]. Other significant operative criteria for our patients included worrisome features on ultrasound, clinical suspicion and patient preference [8]. In addition, diagnostic surgery was recommended to patients with two consecutive diagnoses of B3 or B4 on p-USFNA and r-USFNA.

All statistical analyses were performed using SPSS (IBM Corp., Armonk, NY). This study was approved by the institutional Ethics Review Board (protocol CR15-20).

\section{Results}

A total of 96 patients were retained in this study (Table 1). The mean age at diagnosis was 50.7 years ( $82.3 \%$ female). Table 1 summarizes the pathological characteristics of the excised nodules. The lesions were categorized according to the final histopathological report as benign (40.6\%) or

Table 1 Patient demographics and pathological characteristics of the excised nodules

\begin{tabular}{ll}
\hline Variable & $\begin{array}{l}\text { Participants } \\
(n=96)\end{array}$ \\
\hline Age, years & $50.7(12.2)$ \\
Mean (SD) & $19-75$ \\
Range & \\
Gender, \% & 17.7 \\
Male & 82.3 \\
Female & \\
Nature of lesions, $\mathrm{n}(\%)$ & $39(40.6)$ \\
Benign & $57(59.4)$ \\
Malignant & \\
Type of Lesion, $\mathrm{n}(\%)$ & $56(58.3)$ \\
Papillary Carcinoma & $25(26.0)$ \\
Follicular Adenoma & $15(15.6)$ \\
Other & \\
Histology, $\mathrm{n}(\%)$ & $63(65.6)$ \\
Unifocal lesion & $33(34.4)$ \\
Multifocal lesion & \\
Size of Malignant Lesion, $\mathrm{n}(\%)$ & $17(29.8)$ \\
size $<1$ cm & $21(36.8)$ \\
1 $=<$ size $<2 \mathrm{~cm}$ & \\
$2=<$ size $<4 \mathrm{~cm}$ & \\
size $>=4 \mathrm{~cm}$ & \\
\hline
\end{tabular}


malignant (59.4 \%). Papillary thyroid carcinoma comprised the most common type of histology (58.3\% of all lesions). $56.6 \%$ of nodules were multifocal in histology. In addition, the highest proportion (36.8\%) of malignant thyroid nodules in this study were between 1 and $1.9 \mathrm{~cm}$.

Nodules were reclassified after r-USFNA as suspicious for malignancy or malignant (B5 or B6) in $17.3 \%$ of B3 and $20.0 \%$ of B4 nodules. Moreover, $7.4 \%$ of patients with a score of B3 on p-USFNA had a result of benign lesion (B2) on r-USFNA. The proportion of malignancy on final histopathological reports of lesions categorized as B3 or B4 on primary and repeat USFNAs is shown in Table 2. After p-USFNA, $58.0 \%$ of nodules categorized as B3 and $66.7 \%$ of B4 lesions were found to be malignant. Similarly, $54.6 \%$ of lesions classified as B3 after r-USFNA and $55.7 \%$ of B4 were confirmed to be malignant.

The distribution of Bethesda categories after r-USFNA for lesions initially diagnosed as either B3 or B4 is demonstrated in Table 3, and the corresponding percentage of malignancy of each category is summarized in Table 4.

In addition, differences in the rates of hemi- and completion thyroidectomies between patients with and without a repeat USFNA has been investigated (Table 5). 255 patients had only one USFNA study and did not undergo a repeat procedure; 121 of these patients had a hemi-thyroidectomy operation (47.45\%). Out of the 96 patients who had a repeat USFNA after the diagnosis of B3 or B4 on p-USFNA, 36 underwent hemi-thyroidectomy (37.50\%). Furthermore, 16 out of 255 patients $(6.27 \%)$ without r-USFNA underwent completion thyroidectomy, compared to 3 out of 96 (3.12\%) in patients who obtained a r-USFNA.

\section{Discussion}

Although previous studies have compared the outcomes of repeat USFNAs with the primary ones, post-operative histopathological data has been missing from all the previous similar studies, except one by VanderLaan [1]. This study is the first to examine the significance of repeating USFNA in patients with p-USFNA diagnosis of B4, and to explore the significance of $r$-USFNA in guiding the clinical decision of performing hemi-thyroidectomy versus total thyroidectomy for indeterminate nodules. This study aims to address the gap in the literature with

Table 2 Proportion of malignancy as a function of Bethesda score for $p$-USFNA and r-USFNA

\begin{tabular}{llll}
\hline Diagnostic study & Bethesda score & $\begin{array}{l}\text { Number of } \\
\text { cases (\%) }\end{array}$ & $\begin{array}{l}\text { Malignant final } \\
\text { pathology, n (\%) }\end{array}$ \\
\hline p-USFNA & B3 & $81(84.4)$ & $47(58.0)$ \\
r-USFNA & B4 & $15(15.6)$ & $10(66.7)$ \\
& B3 & $55(73.3)$ & $30(54.6)$ \\
& B4 & $18(26.7)$ & $10(55.7)$ \\
\hline
\end{tabular}

Abbreviations: $p$-USFNA primary fine-needle aspiration, $r$-USFNA Repeat fine-needle aspiration, B3 Bethesda score III, B4 Bethesda score IV
Table 3 Distribution of repeat USFNA cytology according to initial Bethesda classification

\begin{tabular}{|c|c|c|c|c|c|c|}
\hline \multirow[t]{2}{*}{ p-USFNA } & \multicolumn{6}{|c|}{$r$-USFNA } \\
\hline & B2 & B3 & B4 & B5 & B6 & Total \\
\hline \multicolumn{7}{|l|}{$\overline{B 3}$} \\
\hline Frequency & 6 & 50 & 11 & 14 & 0 & 81 \\
\hline$\%$ & 7.4 & 77.2 & 13.6 & 17.3 & 0 & \\
\hline \multicolumn{7}{|l|}{ B4 } \\
\hline Frequency & 0 & 5 & 7 & 1 & 2 & 15 \\
\hline$\%$ & 0 & 33.3 & 46.7 & 6.7 & 13.3 & \\
\hline Total & & & & & & 96 \\
\hline
\end{tabular}

Abbreviations: $p$-USFNA primary fine-needle aspiration, $r$-USFNA Repeat fine-needle aspiration, $B 2$ Bethesda score II, $B 3$ Bethesda score III, $B 4$ Bethesda score IV, B5 Bethesda score V, B6 Bethesda score VI

regards to correlation of results of repeat USFNA and final surgical pathology. However, there exists a trade off between having definite histopathological data and selecting a well-representative sample from the patient population. Necessitating previous hemi- or total thyroidectomy, as an inclusion criterion in this study, may act to introduce a source of selection bias.

Bethesda categories B3 and B4 comprise a variety of heterogeneous lesions that are neither benign (category B2) nor sufficiently atypical for making a diagnosis of suspicious (category B5) or malignant (category B6) lesions [9-11]. Thus, there is great variability in the literature as to whether patients with such indeterminate diagnoses should undergo surgery or not. Previous investigations have demonstrated that $r$-USFNA increases the rate of reclassification of indeterminate nodules into one of the more definitive categories of BSRTC and a higher percentage diagnosis of malignancy (categories B5 or B6) [1, 2, 5, 6, 12-15]. However, there exists great variation surrounding the extent of such reclassification in the literature. The percentage of B3 lesions on p-USFNA that upgrade into a more definitive category of B5 or B6 upon performing r-USFNA ranges from 1.35 to $24.77 \%$ with an average of $11.03 \%$ of cases

Table 4 Percent malignancy of individual Bethesda scores after repeat USFNA, as compared with initial USFNA

\begin{tabular}{llll}
\hline p-USFNA & r-USFNA & Number of cases (\%) & $\begin{array}{l}\text { Malignant final } \\
\text { pathology, } \mathrm{n}(\%)\end{array}$ \\
\hline B3 & B2 & $6(7.4)$ & $0(0.0)$ \\
$(n=81)$ & B3/B4 & $61(75.3)$ & $33(54.01)$ \\
& B5/B6 & $14(17.3)$ & $14(100.0)$ \\
B4 & B2 & $0(0)$ & $0(\mathrm{NA})$ \\
$(n=15)$ & B3/B4 & $12(80)$ & $7(58.33)$ \\
& B5/B6 & $3(20)$ & $3(100.0)$ \\
\hline
\end{tabular}

Abbreviations: $p$-USFNA primary fine-needle aspiration, $r$-USFNA Repeat fine-needle aspiration, $B 2$ Bethesda score II, $B 3$ Bethesda score III, B4 Bethesda score IV, B5 Bethesda score V, B6 Bethesda score VI 
Table 5 Frequency and percentage of hemi- and completion thyroidectomy operations in patients with one or two USFNA diagnostic studies, and a score of B3 or B4 on the initial USFNA

\begin{tabular}{llll}
\hline $\begin{array}{l}\text { Diagnostic } \\
\text { study }\end{array}$ & $\begin{array}{l}\text { Hemi-thyroidectomy, } \\
\mathrm{n}(\%)\end{array}$ & $\begin{array}{l}\text { Completion } \\
\text { thyroidectomy, } \\
\mathrm{n}(\%)\end{array}$ & $\begin{array}{l}\text { Total number of } \\
\text { operated cases }\end{array}$ \\
\hline $\begin{array}{l}\text { p-USFNA } \\
\text { (B3 or B4) }\end{array}$ & $121(47.45)$ & $16(6.27)$ & 255 \\
$\begin{array}{l}\text { p-USFNA } \\
\text { (B3 or B4) }+ \\
\text { r-USFNA }\end{array}$ & $36(37.50)$ & $3(3.12)$ & 96 \\
\hline
\end{tabular}

Abbreviations: $p$-USFNA primary fine-needle aspiration, $r$-USFNA Repeat fine-needle aspiration, $B 3$ Bethesda score III, B4 Bethesda score IV

$[6,12,15-21]$. Our data shows that performing a second USFNA results in an upgrade from indeterminate to more definitive categories of Bethesda classification in approximately $20 \%$ of patients, hence changing their clinical management. Specifically, as a result of r-USFNA, surgical intervention was chosen over clinical follow up in 17.3 and $20.0 \%$ of patients with initial BSRTC score of B3 and B4 respectively.

The efficacy of r-USFNA in avoiding a completion thyroidectomy has also been assessed in this study. Diagnostic hemi-thyroidectomy for B3 nodules is not uncommon, and similarly, BSRTC recommends performing lobectomy for B4 lesions. However, our data demonstrates that $r$ USFNA facilitates choosing the correct surgery (i.e. total thyroidectomy) in about $20 \%$ of nodules that were upgraded from B3/B4 to a more definite malignant category. In these patients, diagnostic hemi- and therapeutic completion thyroidectomies could be avoided. The same concept applies for patients downgraded to a benign diagnosis. In this investigation, $7.4 \%$ of nodules downgraded from B3 to a benign category of B2 after repeating the USFNA. In this group of patients, the clinical management would have changed to a more conservative one not warranting surgery, had they not had a high MTNS score. The proportion of cases in our study that downgraded to a benign score of B2 after r-USFNA are not consistent with those reported in the literature however, ranging from 42.3 to $78.4 \%$ of cases (average $60.3 \%$ ) [6, 16-18, $20,21]$. The reason for this discrepancy could be attributed to the very small number of samples in our study that had a repeat USFNA score of B2. In this study, repeating USFNA has been proven to be effective in identifying nodules that are malignant and suspicious for malignancy to undergo total thyroidectomy, but not in ruling-out surgery for benign nodules upon r-USFNA. The reason behind such difference could be due to the fact that all patients in this study had met operative criteria, based on their MTNS score.

In brief, although as per latest guidelines published by the American Thyroid Association, hemi-thyroidectomy may be beneficial in definitive histological diagnosis of indeterminate nodules, repeat USFNA could lead to a clarity of diagnosis preventing the need for lobectomy [22]. In specific, repeat USFNA of an indeterminate nodule may lead to a change to a more conservative management of a benign nodule or upstaging and total thyroidectomy for a malignant lesion. These results have potential implications for cost reduction in the health care system, prevention of patient work absenteeism, and the avoidance of repeat anesthesia.

The analysis of our thyroidectomy database revealed that performing a second USFNA biopsy decreased the number of hemi-thyroidectomy procedures by $9.95 \%$ and prevented a second surgery in approximately $3 \%$ of cases. The discrepancy between the predicted (20\%) and actual (3\%) rate of reduction in completion thyroidectomy surgeries could be attributed to the fact that many of our patients with one USFNA result of B3/B4 underwent total thyroidectomy in the context of a high MTNS score and increased clinical suspicion of malignancy.

Although no data in the literature compared changes in BSRTC categories after repeating USFNA in nodules initially classified as B4, our results for B3 cases on pUSFNA are consistent with the values reported in similar investigations. Specifically, our results show that the proportion of nodules that were reclassified from B3 into B5/B6 after r-USFNA are similar to those reported by VanderLaan (17.8\%), Chen (15.4\%), Yassa (14.16 \%) and Baloch $(19.42 \%)$ [16, 17, 21, 23].

Our results demonstrate a greater rate of malignancy than what is reported in the BSRTC classification. According to the BSRTC, the risk of malignancy is reported to be $5-15 \%$ for a diagnosis of B3, and 15-30\% for B4 [9]. However, a systematic review has demonstrated a large variation in the risk of malignancy associated with B3 classification, ranging from 6 to $96.7 \%$ (average of $32.7 \%$ ) [3, 14, 15, 23]. Likewise, similar issues occur for the B4 classification. In our study, the overall risk of malignancy, as confirmed by the histopathological data, for a thyroid nodule classified as B3 or B4 on p-USFNA was higher than previous reports in the literature, with $58.0 \%$ of B3 and $66.7 \%$ of B4 nodules confirmed to be malignant $[1-5,11]$. This difference could be explained by the fact that all the patients included in our study had undergone either hemi- or full thyroidectomy procedure, potentially leading to a selection bias. Furthermore, the heterogeneous nature of B3 and B4 categories and disparities in their interpretations could be considered another factor that has led to the higher rates of malignancies in this study.

The decision to perform surgery in our patient population was not solely limited to the results of the USFNA, but was also influenced by a high MTNS score. MTNS portrays a more accurate account of risk of malignancy by taking into consideration salient factors such as 
patient demographics, family history and previous radiation exposure. It also incorporates adverse features on ultrasound and on PET scans as an adjunct to the cytological properties of nodules. Thus, an elevated clinical suspicion of malignancy as suggested by the MTNS score, in conjunction with patient and physician preferences, comprise the operative criteria in our institution and guide the clinical decision making.

There are a number of limitations to this study. First, since all of the patients in this investigation have undergone surgery, a selection bias may explain the higher calculated risk of malignancy of B3/B4 nodules. Other limitations include the retrospective nature of the study and the small number of samples that have a repeat USFNA result consistent with a benign nodule (B2).

\section{Conclusions}

Repeating USFNA in patients with indeterminate diagnoses (B3 or B4) increases categorization into more definite diagnostic categories. Close to $20 \%$ of patients are found to have malignant or suspicious thyroid nodules upon repeating the biopsy, hence a diagnostic hemithyroidectomy was avoided and a more definitive surgery could be performed. Furthermore, repeat USFNA results in a fewer number of hemi-thyroidectomy and completion thyroidectomy procedures.

\section{Abbreviations}

B2: Bethesda score II; B3: Bethesda score III; B4: Bethesda score IV; B5: Bethesda score V; B6: Bethesda score VI; BSRTC: Bethesda System for Reporting Thyroid Cytopathology; p-USFNA: Primary Fine Needle Aspiration; r-USFNA: Repeat Fine Needle Aspiration; USFNA: Ultrasound Guided Fine Needle Aspiration

\section{Funding}

No funding has been provided for this research study.

\section{Availability of data and materials}

The dataset supporting the conclusions of this article is available in the FigShare repository (DOI: 10.6084/m9.figshare.3382846) at https://figshare.com/s/661d890 ab3d30ac3abb5.

\section{Authors' contributions}

All authors have materially participated in the article preparation, and all have approved the final article.

\section{Competing interests}

The authors declare that they have no competing interests.

\section{Ethics approval and consent to participate}

Ethics approval has been obtained from the research ethics committee at the Jewish General Hospital, Montreal, Canada for use of human data through retrospective chart review CR15-20. No individual person's data has been published in this study.

\section{Disclosure}

Nothing to disclose. All authors have materially participated in the article preparation, and all have approved the final article.

\section{Author details}

'Department of Otolaryngology - Head and Neck Surgery - Jewish General Hospital, McGill University, 3755 Chemin de la Côte-Sainte-Catherine, Room E-903, Montréal H3T 1E2, QC, Canada. ²Division of Endocrinology, Jewish
General Hospital - McGill University, Montreal, QC, Canada. ${ }^{3}$ Department of Pathology, Jewish General Hospital - McGill University, Montreal, QC, Canada.

Received: 1 June 2016 Accepted: 10 October 2016

Published online: 18 October 2016

\section{References}

1. VanderLaan PA, Marqusee E, Krane JF. Clinical outcome for atypia of undetermined significance in thyroid fine-needle aspirations: should repeated fna be the preferred initial approach? Am J Clin Pathol. 2011;135:770-5.

2. Hyeon J, Ahn S, Shin JH, Oh YL. The prediction of malignant risk in the category "atypia of undetermined significance/follicular lesion of undetermined significance" of the Bethesda System for Reporting Thyroid Cytopathology using subcategorization and BRAF mutation results. Cancer Cytopathol. 2014;122:368-76.

3. Kholova I, Ludvikova M. Thyroid atypia of undetermined significance or follicular lesion of undetermined significance: an indispensable bethesda 2010 diagnostic category or waste garbage? Acta Cytol. 2014;58:319-29.

4. Mathur A, Najafian A, Schneider EB, Zeiger MA, Olson MT. Malignancy risk and reproducibility associated with atypia of undetermined significance on thyroid cytology. Surgery. 2014;156:1471-6. discussion6.

5. Sullivan PS, Hirschowitz SL, Fung PC, Apple SK. The impact of atypia/follicular lesion of undetermined significance and repeat fine-needle aspiration: 5 years before and after implementation of the Bethesda System. Cancer Cytopathol. 2014;122:866-72

6. Wong LQ, LiVolsi VA, Baloch ZW. Diagnosis of atypia/follicular lesion of undetermined significance: An institutional experience. Cyto J. 2014;11:23.

7. Varshney R, Forest VI, Mascarella MA, et al. The Mcgill thyroid nodule score inverted question mark does it help with indeterminate thyroid nodules? J Otolaryngol. 2015;44:2.

8. American Thyroid Association Guidelines Taskforce on Thyroid N, Differentiated Thyroid C, Cooper DS, et al. Revised American Thyroid Association management guidelines for patients with thyroid nodules and differentiated thyroid cancer. Thyroid. 2009;19:1167-214.

9. Cibas ES, Ali SZ. The Bethesda System for Reporting Thyroid Cytopathology. Thyroid. 2009;19:1159-65.

10. Crippa S, Mazzucchelli L, Cibas ES, Ali SZ. The Bethesda System for reporting thyroid fine-needle aspiration specimens. Am J Clin Pathol. 2010;134:343-4. author reply 5.

11. Horne MJ, Chhieng DC, Theoharis C, et al. Thyroid follicular lesion of undetermined significance: Evaluation of the risk of malignancy using the two-tier sub-classification. Diagn Cytopathol. 2012;40:410-5.

12. Dincer N, Balci S, Yazgan A, et al. Follow-up of atypia and follicular lesions of undetermined significance in thyroid fine needle aspiration cytology. Cytopathol. 2013;24:385-90.

13. Gerhard R, Boerner SL. Evaluation of Indeterminate Thyroid Cytology by Second-Opinion Diagnosis or Repeat Fine-Needle Aspiration: Which Is the Best Approach? Acta Cytologica. 2015;59(1):43-50.

14. Gocun PU, Karakus E, Bulutay P, Akturk M, Akin M, Poyraz A. What is the malignancy risk for atypia of undetermined significance? Three years' experience at a university hospital in Turkey. Cancer Cytopathol. 2014;122:604-10.

15. Ho AS, Sarti EE, Jain KS, et al. Malignancy rate in thyroid nodules classified as Bethesda category III (AUS/FLUS). Thyroid. 2014;24:832-9.

16. Yassa L, Cibas ES, Benson CB, et al. Long-term assessment of a multidisciplinary approach to thyroid nodule diagnostic evaluation. Cancer. 2007:111:508-16.

17. Vanderlaan PA, Krane JF, Cibas ES. The frequency of 'atypia of undetermined significance' interpretations for thyroid fine-needle aspirations is negatively correlated with histologically proven malignant outcomes. Acta Cytol. 2011:55:512-7.

18. Graciano AJ, Chone CT, Fischer CA, Bublitz GS, Peixoto AJ. Repeated fine-needle aspiration cytology for the diagnosis and follow-up of thyroid nodules. Braz J Otorhinolaryngol. 2014;80:422-7.

19. Faquin WC, Baloch ZW. Fine-needle aspiration of follicular patterned lesions of the thyroid: Diagnosis, management, and follow-up according to National Cancer Institute (NCI) recommendations. Diagn Cytopathol. 2010;38:731-9.

20. Chen JC, Pace SC, Chen BA, Khiyami A, McHenry CR. Yield of repeat fineneedle aspiration biopsy and rate of malignancy in patients with atypia or follicular lesion of undetermined significance: the impact of the Bethesda System for Reporting Thyroid Cytopathology. Surgery. 2012;152:1037-44. 
21. Baloch Z, LiVolsi VA, Jain P, et al. Role of repeat fine-needle aspiration biopsy (FNAB) in the management of thyroid nodules. Diagn Cytopathol. 2003;29:203-6.

22. Haugen BR, Alexander EK, Bible KC, et al. 2015 American Thyroid Association Management Guidelines for Adult Patients with Thyroid Nodules and Differentiated Thyroid Cancer: The American Thyroid Association Guidelines Task Force on Thyroid Nodules and Differentiated Thyroid Cancer. Thyroid. 2016;26:1-133.

23. Chen AY, Jemal A, Ward EM. Increasing incidence of differentiated thyroid cancer in the United States, 1988-2005. Cancer. 2009;115:3801-7.

Submit your next manuscript to BioMed Central and we will help you at every step:

- We accept pre-submission inquiries

- Our selector tool helps you to find the most relevant journal

- We provide round the clock customer support

- Convenient online submission

- Thorough peer review

- Inclusion in PubMed and all major indexing services

- Maximum visibility for your research

Submit your manuscript at www.biomedcentral.com/submit 